\title{
Lymphogranulomatosis Progress in Patient with EBV Infectious Mononucleosis
}

\author{
DOI: $10.17691 / \mathrm{stm} 2015.7 .3 .22$ \\ Received October 16, 2014
}

\author{
T.V. Stchuklina, Physician"; \\ O.A. Saburova, Physician'; \\ I.A. Otmachova, Tutor, Department of Infectious Diseases; \\ O.V. Korochkina, MD, DSc, Professor, Head of the Department of Infectious Diseases²; \\ D.M. Sobchak, MD, DSc, Professor, Department of Infectious Diseases ${ }^{2}$
}

\begin{abstract}
${ }^{1}$ Clinical Infectious Hospital No.9, 1 Ukrainskaya St., Nizhny Novgorod, 603010, Russian Federation; ${ }^{2}$ Nizhny Novgorod State Medical Academy, 10/1 Minin and Pozharsky Square, Nizhny Novgorod, 603005, Russian Federation
\end{abstract}

There has been presented a case history of lymphogranulomatosis in a patient with EBV infectious mononucleosis, which confirms possible activation of lymphoproliferative diseases and hematological malignancies after acute EBV infection, and proves the need for a wide use of virological and immunological techniques.

Key words: herpes viruses; Epstein-Barr virus; immune response; lymphogranulomatosis.

The Epstein-Barr virus (EBV) exhibits a marked tropism towards B-lymphocytes, where it proliferates, T-cells and lymphadenoids. Unlike other herpes viruses, it promotes proliferation of affected cells (generalized lymphadenopathy). EBV is one of oncogenic DNA-containing viruses and acts as an etiological agent for Burkitt lymphoma, nasopharyngeal carcinoma, some forms of lymphogranulomatosis, autoimmune diseases, etc.

Incubation period lasts 4 to 14 days, more seldom up to a month. Very often acute onset is reported: rise of body temperature, headache, muscular and joint pains. Patients suffer from soreness in the throat, the pain growing when swallowing, difficult nasal breathing, hyperplasia of lymphatic nodes. Intensity of clinical symptoms grows by day $4-6$, but already by day $3-4$ hepatolienal syndrome is observed, atypical mononuclear cells are found in the blood. The main symptoms are fever, tonsillitis, generalized lymphadenopathy, hepatosplenomegaly, exanthema (macular, roseolous, papular, etc.). Mandibular and posterior cervical lymph nodes are affected more often, while axillary, inguinal and cubital ones are less often involved. In some patients rather evident picture of acute mesenteric lymphadenitis (mesadenitis) can be observed.

Specific changes in the peripheral blood occur after day 5 of the disease: leukocytosis is developing (hyperleukocytosis is possible, $\left.(18-20) \cdot 10^{9} / \mathrm{L}\right)$, lymphomonocytosis, the number of plasmocytes is increasing, atypical mononuclear cells are emerging (10-60\% and higher). Recovery occurs in 2-4 weeks but lymphadenopathy, hepatosplenomegaly, atypical mononuclear cells may persist, which indicates prolonged disease progression.

There are several possible outcomes of the acute infectious process: recovery, asymptomatic viruscarriage or latent infection, chronic recurrent infection, chronic fatigue syndrome, development of an autoimmune disease and an oncological (lymphoproliferative) process (lymphogranulomatosis, multiple polyclonal lymphomas, nasopharyngeal carcinoma, leukoplakia of the tongue and oral cavity mucous membranes, gastrointestinal cancer, etc.).

Lymphogranulomatosis is a malignant lymphoid tissue

For contacts: Sobchak Devora Mikhailovna, e-mail: sobchak_devora@mail.ru 
neoplasm characterized by its granulomatous structure with presence of giant Reed-Sternberg cells, lesion of lymph nodes and viscera.

The microscopic picture of lymphogranulomatosis is characterized by growth of lymphoid cells and plasmocytes, histiocytes, fibroblasts, neutrophil leukocytes, similar to granulomatous tissue. In the course of granulomatosis, giant Reed-Sternberg cells are found, whose specific features are giant-sized cytoplasm and nuclei; signs of nuclear division without cytoplasm division; large nucleoli resembling intranuclear inclusions; cell nuclei with a delicate chromatin network thickening to the periphery, which creates a clearing area in the centre of the nucleus.

Here is a clinical case that confirms the possibility of developing lymphoproliferative diseases and hematological malignancies after acute EBV infection.

Patient gave written informed consent for the scientific analysis of their data.

Patient K., 24 years old. On admission the patient complained of soreness in the throat and pain when swallowing. He had been ill for a month: high temperature persisted for two days $\left(38-39^{\circ} \mathrm{C}\right)$, soreness in the throat and pain when swallowing occurred a few days later, a week before the admission to hospital supraclavicular and neck lymphatic glands enlarged.

On admission the patient's condition was estimated as satisfactory, the skin was of normal color. On palpation supraclavicular and cervical glands appeared to be enlarged up to $1.5-2 \mathrm{~cm}$, painful, with a dense-elastic texture (Figure 1). Axillary and inguinal glands were also enlarged $(0.5-1 \mathrm{~cm})$, painless, with a soft-elastic texture. In the oropharyngeal cavity there was hyperemia and hypertrophy of the tonsils, the lacunae were covered with purulent deposits (Figure 2). The liver was enlarged, protruded by $2-3 \mathrm{~cm}$ below the costal margin and had a soft-elastic texture, the lower spleen pole was palpated (by percussion, in the $9-11^{\text {th }}$ intercostal space).

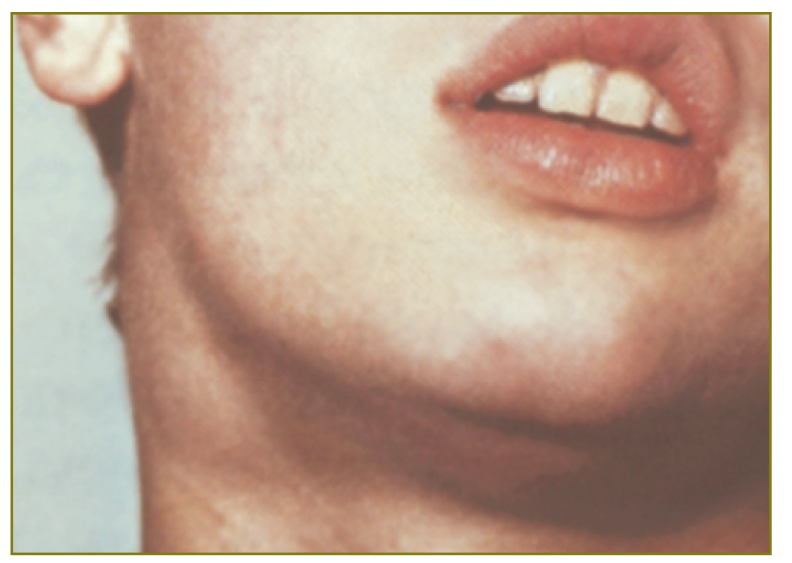

Figure 1. The enlargement of neck lymphatic glands in a patient with EBV infectious mononucleosis
Complete blood count: $\mathrm{Hb}-128 \mathrm{~g} / \mathrm{L} ; \mathrm{RBC}-4.0 \cdot 10^{12} / \mathrm{L}$; color index - 0.96; ESR - $11 \mathrm{~mm} / \mathrm{h} ; W B C-6.4 .10^{\circ} / \mathrm{L}$; segmented neutrophils - 75\%; EOS - 3\%; LYM - 18\%; MON - 4\%. Total bilirubin - $20(5 / 15) \mathrm{mmol} / \mathrm{L} ; A L T-$ $0.6 \mu \mathrm{mol} / \mathrm{L} \cdot \mathrm{h}$.

EBV DNA was found in the blood leukocytes and in the mucous membrane scraping from the oropharyngeal cavity by polymerase chain reaction method. HIV infection markers were negative.

Abdominal ultrasound examination revealed several round-shaped hypoechoic foci with irregular contours $(5 \times 6 \times 7 \mathrm{~mm})$ in the spleen.

The patient was consulted in the Oncologic Dispensary. Findings of the puncture biopsy of the left supraclavicular gland showed lymphoid tissue hyperplasia of infectiousallergic genesis. Examination by phthisiatrician did not reveal any elements of specific inflammation and malignant neoplasm. Hematologist diagnosed no blood disorders at the time of examination on the basis of the clinical laboratory findings.

On the basis of clinical laboratory and instrumental studies moderately severe EBV infectious mononucleosis was diagnosed. The patient received symptomatic and pathogenic therapy: Oxamp (7 days), Cycloferon (14 days), Diazolin, ascorbic acid, calcium chloride. General state of the patient improved. He was discharged from the hospital with positive dynamics (the temperature returned to normal, the lymphatic glands decreased in size).

Six months later again there was reported rise in temperature, enlargement of the axillary and cervical lymphatic glands. The patient was hospitalized to the Oncologic Dispensary. Repeated biopsy of the supraclavicular gland was performed. On histological bioptate examination there was revealed growth of lymphoid cells and plasmocytes, histiocytes, fibro-blasts and neutrophil leukocytes. Secondary to granulomatosis, giant Reed-Sternberg cells were found (giant-sized cytoplasm and nuclei; signs of nuclear division without cytoplasm division; cell nuclei with delicate chromatin

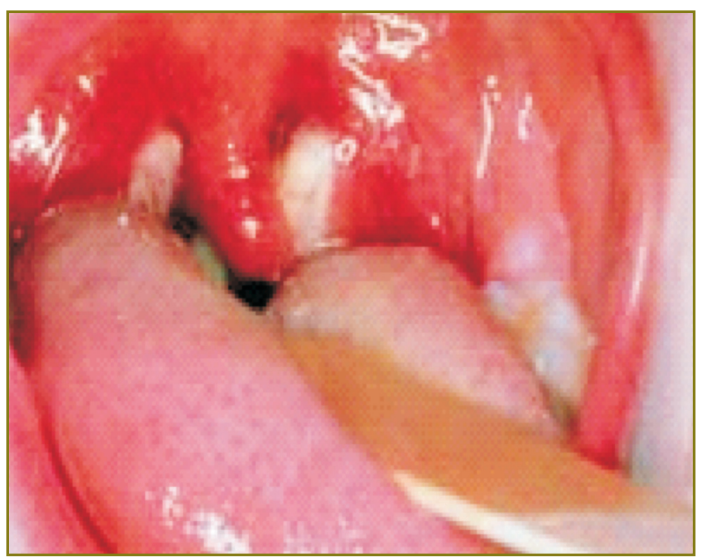

Figure 2. Tonsillar syndrome in a patient with EBV infectious mononucleosis 
network thickening to the periphery). Final diagnosis: "lymphogranulomatosis, stage II, with supraclavicular and axillary lymphatic glands involvement".

Follow-up and further treatment of the patient was carried out in the Oncologic Dispensary.

In the observation described above, lymphogranulomatosis can be estimated as an outcome of EBV infectious mononucleosis. Therefore, for accurate diagnosis and appropriate prediction of the EBV infection outcomes it is necessary to take into account and properly assess a wide variety of clinical symptoms and biochemical, immunological and morphological findings.

Study Funding. This study was not supported by any financial sources.

Conflicts of Interest. The authors have no conflict of interests to disclose. 